\title{
ON A LOCALLY COMPACT GROUP ACTING ON A MANIFOLD
}

\author{
PAUL S. MOSTERT ${ }^{1}$
}

Introduction. Let $G$ be a locally compact group, admissibly topologized [5], operating as a transitive transformation group on a manifold $M$. Using the notation of [1], let $G_{x}$ be the subgroup of $G$ which leaves the point $x$ fixed. Montgomery [1] has proved the following result.

TheOREM (MONTGOMERy). If $G$ is a connected Lie group which acts transitively on a compact manifold $M$, and if $G_{x}$ is connected, then $G$ contains a compact subgroup which acts transitively on $M$.

The purpose of this note is to show that the theorem is true when $G$ is any locally compact group satisfying the second axiom of countability. It will follow, by [4], that the corollaries of [1] are true for the more general case.

1. A lemma. Arens [5] has proved that $M$ is homeomorphic to $G / G_{x}$. The author $[2 ; 3]$ has proved that $G$ is a fibre bundle over $G / G_{x}$.

Lemma. Let $G$ be a connected locally compact group satisfying the second axiom of countability and acting transitively on a manifold $M$. Then there exists a Lie group $G^{*}$ acting transitively on $M$ such that $G^{*}$ is a factor group of $G$ by a compact normal subgroup of $G_{x}$.

Proof. Let $N$ be a compact normal subgroup of $G$ such that $G / N$ is a Lie group [6], and $N^{*}=N \cap G_{x}$. We shall show that $G / N^{*}=G^{*}$ is a Lie group which acts transitively on $M$. As mentioned above, the author [3] has shown that $G / N^{*}$ is a fibre bundle over $G / G_{x}$ with fibre $G_{x} / N^{*}$, and hence $G / N^{*}$ is locally homeomorphic to $\left(G_{x} / N^{*}\right)$ $\times K$ where $K$ is homeomorphic to a euclidean space. Hence, $G / N^{*}$ is a manifold. Now $G / G_{x}$ is homeomorphic and isomorphic to $(G / N) /\left(G_{x} / N^{*}\right)$ so that by a proper choice of $N, \operatorname{dim}\left(N / N^{*}\right)=0$. Since $G / N^{*}$ is a fibre bundle over $G / N, G / N^{*}$ is locally homeomorphic with $\left(N / N^{*}\right) \times L$ where $L$ is a euclidean space. Hence, $N / N^{*}$ is discrete. This implies that a subgroup of $N^{*}$ (which we may assume to be $N^{*}$ itself) has the property that $G / N^{*}$ is a Lie group. Define, for $g^{*} \in G^{*}, g^{*}=g N^{*}, y \in M$,

Received by the editors February 12, 1954. ment.

1 This work was done under Contract N7-Onr-434, Task Order III, Navy Depart- 


$$
g^{*}(y)=g N(y) .
$$

It is not difficult to show that $G^{*}$ forms a transformation group on $M$ with this definition. Since $N^{*} \subset G_{x}, G^{*}$ is transitive.

2. The theorem. Under the hypothesis of the lemma, and if $G_{x}$ is moreover connected, then Montgomery's theorem implies that $G^{*}$ contains a compact subgroup $H^{*}$ which acts transitively on $M$ for $M$ compact. If $p$ is the natural projection $G \rightarrow G^{*}$, then $p^{-1}\left(H^{*}\right)$ $=H$ is compact and acts transitively on $M$. Hence, we have proved the theorem as stated below.

THEOREM. Let $G$ be a connected locally compact group satisfying the second axiom of countability which acts transitively on a compact manifold. If $G_{x}$ is connected, then $G$ contains a compact subgroup which acts transitively on $M$.

\section{BIBLIOGRAPHY}

1. D. Montgomery, Simply connected homogeneous spaces, Proc. Amer. Math. Soc. vol. 1 (1950) pp. 467-469.

2. P. S. Mostert, Local cross sections in locally compact groups, Proc. Amer. Math. Soc. vol. 4 (1953) pp. 645-649.

3. - Sections in principal fibre spaces, to appear.

4. —_, On fibre spaces with 0-dimensional fibres, Duke Math. J. vol. 21 (1954) pp. 67-74.

5. R. Arens, Topologies for homeomorphism groups, Amer. J. Math. vol. 68 (1946) pp. 593-610.

6. H. Yamabe, A generalization of a theorem of Gleason, Ann. of Math. vol. 58 (1953) pp. 351-365.

Tulane, University 